\title{
Hemorragia gastrointestinal superior secundaria a úlceras por estrés en el paciente hospitalizado
}

\author{
Upper gastrointestinal bleeding related to stress ulcers in the hospitalized \\ patient
}

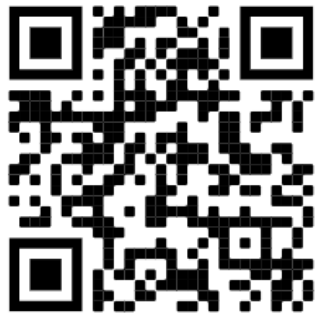

Recibido 07/11/2020

\author{
${ }^{1}$ Dra. Daniela González Jiménez \\ Investigadora independiente, San José, Costa Rica. \\ (iD) https://orcid.org/0000-0002-3514-0703 \\ ${ }^{2}$ Dra. Susan Mejía Bonilla \\ Investigadora independiente, San José, Costa Rica. \\ (iD) https://orcid.org/0000-0003-1570-5962 \\ ${ }^{3}$ Dra. Marisol Cruz Fallas \\ Investigadora independiente, San José, Costa Rica. \\ (iD) https://orcid.org/0000-0001-8065-5506
}

Corregido

$19 / 11 / 2020$

Aceptado

$29 / 01 / 2021$

\section{RESUMEN}

Las úlceras por estrés se definen como erosiones de la mucosa gástrica, originadas por el desbalance fisiológico que se desencadena debido a patologías severas. Algunos de los mecanismos que contribuyen a su origen son el exceso en la producción de endotelina-1, déficit de óxido nítrico, hipoperfusión sistémica, estimulación producción de gastrina y por ende ácido clorhídrico, déficit prostaglandinas y disfunción de esfínteres. Esta fisiopatología se presenta comúnmente en pacientes gravemente enfermos, por lo cual es una patología que debe sospecharse en usuarios de la Unidad de Cuidados Intensivos. El uso de anticoagulantes orales y antiinflamatorios no esteroideos, ventilación mecánica asistida, traumas severos, sepsis y enfermedad renal o hepática crónica, son algunos de los factores de riesgo más comunes que favorecen la aparición de esta enfermedad. Las úlceras por estrés tienden a ser superficiales y de bordes definidos. La clínica presentada en las úlceras gástricas por estrés puede ir desde pacientes asintomáticos hasta pacientes con sangrados profusos e inestabilidad hemodinámica. Se clasifica a los pacientes en cuatro categorías, estas dependiendo del riesgo de origen de úlceras que se presente; bajo, moderado, alto y muy alto riesgo. Según la estratificación del paciente, se recomienda el inicio de terapia profiláctica con medicamentos inhibidores de la producción de ácido clorhídrico como Inhibidores de la bomba de protones y antagonistas de los receptores de histamina,

\footnotetext{
${ }^{1}$ Médica general, graduada de la Universidad de Iberoamérica (UNIBE). cod. MED16704, correo: daniigonzalez23@gmail.com

${ }^{2}$ Médica general, graduada de la Universidad Autónoma de Centro América (UACA), cod. MED16507, correo: sg.mejia@hotmail.com

${ }^{3}$ Médica general, graduada de la Universidad Autónoma de Centro América (UACA), cod. MED15494, correo: dracruzf@gmail.com
} 
siendo demostrado su beneficio en la morbi/mortalidad de estos pacientes.

PALABRAS CLAVE: ácido gástrico; mucosa gástrica; úlcera gástrica; úlcera péptica perforada; hemorragia gastrointestinal; estrés fisiológico; inhibidores de la bomba de protones.

\section{ABSTRACT}

Stress ulcers are defined as erosions of the gastric mucosa, caused by physiological imbalances that are triggered by severe symptoms. Pathophysiology of stress ulcers originate from endothelin-1 excess production, nitric oxide deficiency, systemic hypoperfusion, hyperstimulation of gastrin production and therefore production of hydrochloric acid, prostaglandin deficiency and sphincter dysfunction. This pathophysiology occurs in seriously ill patients, which is why it can be found in patients admitted to an Intensive Care Unit. The use of oral anticoagulants and non-steroidal anti-inflammatory drugs, assisted mechanical ventilation, severe trauma, sepsis, and chronic kidney or liver disease are some of the most common risk factors to the development of this disease. Stress ulcers are superficial and are defined by a visible border. Patients can be asymptomatic or display a variety of symptoms as far as profuse bleeding and hemodynamic instability. Patients are classified into four categories according to the severity of risk factors; low, moderate, high, and very high risk of stress ulcers. Depending on the category of risk that the patient presents, it is recommended to start prophylactic therapy with drugs that inhibit the production of hydrochloric acid such as Proton Pump Inhibitors and Histamine Receptor Blockers. This medications have shown their benefits by reducing the mobility and mortality rates of stress ulcers patients.

KEYWORDS: gastric acid; gastric mucosa; stomach ulcer; peptic ulcer perforation; peptic ulcer hemorrhage; physiological stress; proton pump inhibitors.

\section{INTRODUCCIÓN}

Las úlceras por estrés se definen como erosiones de la mucosa gástrica originadas por eventos estresantes fisiológicamente. Estos eventos pueden ser quemaduras extensas, ventilación mecánica asistida, cirugía mayor, sepsis, coagulopatías o traumatismo grave.

La mayoría de las úlceras por estrés son superficiales y asintomáticas, pero la ulceración puede progresar hasta erosionar vasos más grandes, resultando en un sangrado gastrointestinal manifiesto (SGI) (1).

EI SGI representa una de las condiciones clínicas más prevalentes en los Servicios de Urgencias y Gastroenterología, generando una morbilidad y mortalidad de importancia (2).
La profilaxis se prescribe a pacientes hospitalizados que corren moderado, alto y muy alto riesgo de sangrado por lesiones gastroduodenales, y a pacientes que presentan enfermedad previamente asintomática que ha sido desenmascarada por la hospitalización (ㅁ) .

Los medicamentos supresores de ácido clorhídrico $(\mathrm{HCl})$, incluidos los inhibidores de la bomba de protones (IBP), los antagonistas de los receptores $\mathrm{H} 2$ (anti $\mathrm{H} 2$ ) y los protectores de la mucosa gástrica, son las opciones más recomendadas para la profilaxis de las úlceras por estrés en pacientes críticos con factores de riesgo.

La siguiente revisión bibliográfica tiene como enfoque principal demostrar el conjunto de medidas que se pueden implementar para la prevención del Sangrado Digestivo Alto (SDA) secundario a lesión mucosa 
relacionada al estrés (LMRS) en el paciente hospitalizado.

\section{MÉTODO}

El presente artículo es la presentación de una revisión bibliográfica, para la cual se revisaron variadas fuentes de investigación científica. Se utilizaron bases de datos como PubMed, ScienceDirect, Cochrane, UpToDate y Scielo, con bibliografía tanto en inglés como en español. Además, se filtró la búsqueda con el fin de obtener artículos menores a cinco años de antigüedad, comprendidos entre el año 2015-2020. Para su realización se tomaron de referencia cuatro revistas científicas, entre ellas destacar The New England Journal of Medicine y Current Opinion in Gastroenterology.

Se consultó un libro y diez artículos digitales tomados de internet para la recolección de la información requerida. Se excluyeron aquellos artículos que estuvieran publicados en idiomas diferentes al inglés o español, o que presentaran una antigüedad mayor a cinco años.

\section{EPIDEMIOLOGÍA}

EI SDA es una complicación infrecuente pero clínicamente significativa en los pacientes hospitalizados. Contribuye sustancialmente a la morbilidad y mortalidad hospitalaria. Afecta mayormente al sexo masculino y la edad avanzada es directamente proporcional al riesgo de hemorragia.

El sangrado gastrointestinal oculto se produce del 15 al $50 \%$ de los pacientes en estado crítico, y la hemorragia evidente se presenta del 5 al $25 \%$ de los pacientes críticos que no recibieron profilaxis previa. (므무).

\section{FISIOPATOLOGÍA}

Las células parietales en la mucosa gástrica, estimuladas por señales neurohormonales, producen $\mathrm{HCl}$.

Este tiene un $\mathrm{pH}$ de 2, esencial para la digestión de alimentos y eliminación de patógenos ingeridos. Aunque este nivel de $\mathrm{pH}$ desintegraría rápidamente la mayoría de los tejidos, son las prostaglandinas y el óxido nítrico los responsables de mantener una capa mucosa que protege el epitelio gástrico.

El flujo sanguíneo normal suministra oxígeno y bicarbonato, los cuales eliminan los iones de hidrógeno que se difunden desde la luz hacia la mucosa gástrica. Múltiples sensores de ácido monitorean el $\mathrm{pH}$ extracelular, lo que puede desencadenar disminución de la producción de gastrina y $\mathrm{HCl}$ si se censa un aumento desmesurado en su cantidad o nivel de acidez (4).

La función coordinada del esfínter esofágico inferior y el esfínter pilórico equilibran el pH en el esófago y el duodeno, los cuales en relación con el estómago, poseen epitelio menos resistente a los ácidos. En conjunto, estos mecanismos crean un sistema de defensa crucial para proteger el epitelio gastrointestinal.

La ulceración de la mucosa gástrica resulta del desbalance que se genera en su sistema de protección debido a estrés sistémico. Esto da como resultado una exposición desmesurada de la mucosa al $\mathrm{HCl}$, propiciando múltiples erosiones superficiales de la misma.

Se cree que este desbalance se debe a alteraciones en las glucoproteínas de la mucosa, las cuales son denudadas por concentraciones elevadas de sales biliares 0 toxinas urémicas, y por reflujo debido a enfermedad crítica. 
Otro factor que se plantea es el aumento de la secreción de $\mathrm{HCl}$ en respuesta a una mayor secreción de hormona gastrina, la cual se estimula bajo la presencia de un evento estresante fisiológicamente ( $\underline{5})$.

En la mayoría de los pacientes críticamente enfermos, el flujo sanguíneo de la mucosa gástrica está alterado.

Las razones incluyen cambios hemodinámicos sistémicos (hipotensión y/o terapia vasopresora) y/o alteraciones locales. Por ejemplo, flujo sanguíneo esplácnico reducido debido a presión positiva al final de la espiración en pacientes ventilados mecánicamente (므). Además del daño tisular isquémico en sí, la hipoperfusión conduce a una producción reducida de varios mecanismos protectores como la producción de moco, fosfolípidos, bicarbonato y proteínas de choque térmico.

Por otro lado, dos importantes reguladores moleculares de la tensión vascular están desregulados en enfermedades críticas. Mientras que la producción del óxido nítrico vasodilatador se reduce, el nivel de endotelina-1, un vasoconstrictor fuerte, aumenta significativamente.

Este cambio puede contribuir a dañar aún más la mucosa. Si bien estos mecanismos pueden causar daño al recubrimiento gastrico, a menudo son insuficientes por sí mismos para causar ulceraciones importantes y SDA.

El componente crucial para el daño manifiesto es la presencia de $\mathrm{HCl}$. Sin ácido clorhídrico, el daño de la mucosa suele ser subclínico. Esto proporciona la justificación para el uso de fármacos supresores de ácido como PPI o H2RA para la profilaxis farmacológica $(7, \underline{8})$.

\section{FACTORES DE RIESGO}

Los factores de riesgo más prevalentes para el SDA secundario a LMRS en pacientes hospitalizados son (ㅁ):

1. Enfermedad aguda: shock, falla respiratoria, trauma craneoencefálico, politrauma, lesión térmica.

2. Condiciones crónicas: disfunción renal, enfermedad hepática, coagulopatías, infección por Helicobacter pylori.

3. Medicamentos: anticoagulantes, antiagregantes plaquetarios, AINES de uso prolongado.

4. Dispositivos: ventilación mecánica asistida, hemodiálisis, soporte vital extracorpóreo.

- Paciente críticamente enfermo: los pacientes críticamente enfermos corren mayor riesgo de desarrollar LMRS. La fisiopatología no se comprende completamente. Se ha planteado la hipótesis de que las úlceras por estrés son causadas por la disminución del flujo sanguíneo de la mucosa, la isquemia y la lesión por reperfusión y, por lo tanto, están menos relacionadas con la secreción de ácido que las úlceras pépticas. La mayoría de las úlceras por estrés son superficiales y asintomáticas, pero la ulceración puede progresar y erosionar vasos más grandes, lo que resulta en una hemorragia gastrointestinal manifiesta (1).

Los dos factores de riesgo principales en estos pacientes son la coagulopatía grave y la ventilación mecánica asistida durante más de 48 horas. La frecuencia de SDA clínicamente significativo con uno o ambos factores de riesgo es del $3,7 \%$, en comparación con el $0,1 \%$ cuando ninguno de los factores de riesgo 
está presente. Otros factores de riesgo propuestos incluyen antecedentes de hemorragia $\mathrm{GI}$, sepsis, ingreso en $\mathrm{UCl}$ por más de 7 días, hemorragia GI oculta durante más de 5 días y tratamiento con dosis altas glucocorticoides (므).

La lesión neurológica combinada con estrés fisiológico severo (por ejemplo, lesión cerebral traumática) que provoca la admisión a la UCl puede amplificar la probabilidad de hemorragia relacionada con el estrés. Los perfiles de riesgo específicos de la población se basan en la gravedad de las enfermedades agudas y crónicas y en ciertos medicamentos e intervenciones utilizados en el hospital (무).

- Paciente hospitalizado que no está críticamente enfermo: los pacientes que no están críticamente enfermos, independientemente de su ingreso en unidades médicas o quirúrgicas, presentan una incidencia menor que aquellos que están gravemente enfermos en unidades especializadas. Esta incidencia puede diferir dependiendo de las patologías de base que presente el paciente; por ejemplo, en pacientes que presenten una lesión renal aguda, el sangrado puede ser mayor.

La tasa de sangrado clínicamente importante en estos pacientes es de $0,005 \%$.

Los episodios de sangrado se deben principalmente a la enfermedad por úlcera duodenal y suelen presentarse después de un período medio de 14 días de hospitalización (므).

En pacientes ingresados en un servicio de medicina general, los principales factores de riesgo de hemorragia fueron la terapia anticoagulante y el uso de clopidogrel.
Los factores de riesgo independientes para el SDA por LMRE incluyen: edad de más de 60 años, sexo masculino, enfermedad hepática, insuficiencia renal aguda, sepsis, anticoagulación profiláctica y coagulopatía con o sin la administración de agentes antiplaquetarios.

El pronóstico después de un episodio de SDA puede depender tanto de las enfermedades agudas y crónicas y de la cantidad de pérdida de sangre como de la causa del sangrado identificada endoscópicamente. La sepsis, la insuficiencia renal y la cirrosis se asociaron con un mayor riesgo de muerte entre los pacientes que tuvieron un episodio de sangrado (므).

\section{CLÍNICA}

La hemorragia por úlcera gástrica en pacientes hospitalizados generalmente se divide en 2 categorías:

1. Lesión mucosa relacionada con estrés (LMRE o úlceras por estrés), caracterizada por sangrado difuso a partir de erosiones y úlceras superficiales. Esta es la razón más frecuente.

2. La segunda categoría corresponde a las úlceras crónicas, reagudizadas en pacientes hospitalizados. Son úlceras grandes, focales, de aspecto crónico e indoloras. Debutan con hemorragia digestiva alta manifestada por hematoquecia, melena o hematemesis.

La LMRE aparece en el tubo digestivo alto

de pacientes hospitalizados en estado grave. Por lo general, se localiza en el estómago, pero también se puede observar 
en el duodeno, el esófago e, incluso, el recto (ㅁ).

La clínica en general puede manifestarse de forma silente o florida. El paciente puede iniciar con síntomas inespecíficos como dolor abdominal, mareos, aturdimiento y somnolencia; avanzando el cuadro con signos como hematemesis, rectorragia, melena, hematoquecia, criodiaforesis, taquicardia, síncope, hipotensión y shock hipovolémico.

El examen físico debe prestar especial atención a la estabilidad hemodinámica que presente el paciente, la presencia de dolor abdominal o el signo de rebote positivo, tacto rectal y evaluación del color de las heces.

Es importante además realizar estudios de laboratorio como hemograma completo, guayaco, pruebas de función hepática, pruebas de función renal y pruebas de coagulación. La hemoglobina igual o menor a $8 \mathrm{mg} / \mathrm{dl}$ es indicación para iniciar transfusión sanguínea posterior a la confirmación de sangrado gastrointestinal (10).

\section{DIAGNÓSTICO}

La esofagogastroduodenoscopia (EGD) es el estudio de imagen más utilizado para confirmar o descartar la patología del tracto gastrointestinal superior. Con esta se puede evidenciar la localización, tamaño y profundidad de las úlceras por estrés, además de brindar control del sangrado activo si este se evidencia durante el estudio. Se debe tener en cuenta que en los pacientes que presentan hematemesis como síntoma principal, la EGD es diagnóstica solamente en el $71 \%$ de los casos (11).

Las opciones de control para el sangrado activo que se pueden ofrecer por medio de la EGD son; coagulación térmica, el uso del hemoclip, inyección local de epinefrina y colocación de agentes tópicos como Endoclot y Hemospray. El uso de terapia dual ha demostrado mayor beneficio que la monoterapia con los procedimientos anteriormente descritos (12).

Otra opción diagnóstica que ha tomado fuerza en los últimos años es la Endoscopia por Video Cápsula (EVC). Esta consiste en un pequeño dispositivo que el paciente deglute y envía imágenes seriadas de todo el sistema gastrointestinal. Se puede utilizar con seguridad para evaluar y visualizar la gravedad y persistencia del sangrado. Es importante destacar que es un estudio más específico que el aspirado nasogástrico, en donde solo se evidencia el sangrado del sistema gastrointestinal superior y no su origen. El costo de la ECV, la disponibilidad del equipo y la falta de experiencia en el uso de este dispositivo, son barreras importantes en la implementación global de la endoscopia con videocápsula (13).

En el paciente con antecedente de sangrado gastrointestinal previo, el cual no ha tenido un óptimo control de su patología de base, se puede evidenciar anemia por deficiencia de hierro y sangre oculta en heces en los exámenes de laboratorio (14). La relación nitrógeno/creatinina puede ayudar a diferenciar el sangrado digestivo alto del sangrado digestivo bajo. Cuando se presenta un valor igual o mayor a 36 , se ha demostrado una sensibilidad del $90 \%$ de origen del sangrado de las vías digestivas altas (14).

\section{TRATAMIENTO}

- Agentes supresores del ácido clorhídrico: los medicamentos supresores de ácido evitan el sangrado a través de diferentes mecanismos. Proporcionan barreras físicas al neutralizar el $\mathrm{HCl}$ y mejoran la protección 
de la mucosa estimulando la liberación de prostaglandinas. Tanto los antihistaminicos $\mathrm{H} 2$ (antiH2) como los inhibidores de bomba de protones (IBP) protegen la mucosa gástrica al reducir la secreción de $\mathrm{HCl}$. Los antiH2 bloquean el receptor $\mathrm{H} 2$ de histamina, que es una de las tres vías que activan la bomba de protones, mientras que los IBP inactivan el paso final en la secreción de $\mathrm{HCl}$ al unirse covalentemente a los residuos de cisteína en la bomba de protones.

En teoría, los IBP proporcionan una supresión de ácido mucho más fuerte que el los antiH2, porque están unidos de manera específica e irreversible a la bomba de protones. Los IBP se asociaron con una incidencia significativamente menor de hemorragia debida a úlceras por estrés en comparación con otros medicamentos supresores de ácido (10).

Para los pacientes de bajo riesgo en $\mathrm{UCl}$, en las unidades médicas o quirúrgicas, y en la comunidad, el uso de supresión de ácido en ausencia de una indicación clara puede acarrear consecuencias graves. Se ha demostrado que los efectos adversos de la supresión de ácido pueden predisponer a los pacientes a infecciones nosocomiales. La evidencia vincula las infecciones y la supresión de $\mathrm{HCl}$ debido a la alteración del microbioma gastrointestinal normal que se presenta al consumir estos medicamentos.

Se favorece un crecimiento anómalo de comunidades bacterianas endógenas o la colonización de bacterias patógenas (ㅁ) .

- Profilaxis del sangrado gastrointestinal: actualmente, existe información controversial en relación con el uso de inhibidores de la producción de $\mathrm{HCl}$, IBP y antiH2, en pacientes hospitalizados en estado no crítico.

Existe poca evidencia con respecto al beneficio de la profilaxis para el sangrado gastrointestinal fuera del entorno de cuidados intensivos. No hay consenso sobre si la profilaxis de ulceras por estrés es realmente efectiva y cual agente es la mejor opción para pacientes que no se encuentran en $\mathrm{UCl}$ (13).

Esto podría explicarse debido a que esta patología se encuentra, con mayor frecuencia, en pacientes en la $\mathrm{UCI}$, los cuales presentan un alto riesgo de SDA por LMRE con el solo hecho de estar admitidos en esta unidad.

La morbilidad del SDA debido a úlceras por estrés varían desde inestabilidad hemodinámica, hipotensión, taquicardia, falla respiratoria, anemia, shock hipovolémico, sepsis y muerte. Esto evidencia, que la consideración y aplicación de tratamiento profiláctico en este grupo de pacientes es de gran importancia (14).

- Profilaxis contra sangrado gastrointestinal en pacientes hospitalizados que no encuentran internados en $\mathrm{UCl}$ : la profilaxis de ulceras por estrés podría ser necesaria en pacientes que no se encuentran en la $\mathrm{UCl}$ pero presentan factores de riesgo que pueden jugar un papel importante en el desarrollo de SDA (13). En este caso, se recomiendan la utilización de agentes supresores de ácido en pacientes con factores de riesgo de SDA, como por ejemplo;

pacientes que requieran terapia antiplaquetaria o anticoagulante, pacientes con antecedente de 
traumatismos graves o antecedente de cirugía mayor ( $\underline{3})$.

Sin embargo, se han realizado pocos ensayos que evalúen la eficacia de la profilaxis contra la úlcera por estrés en pacientes ingresados en unidades médicas y quirúrgicas. En el artículo "Profilaxis contra el sangrado gastrointestinal superior en pacientes hospitalizados", publicado por la Revista Médica de Nueva Inglaterra (NEJM, por sus siglas en inglés) se realizó un estudio observacional que involucró a 63.878 pacientes hospitalizados que no estaban críticamente enfermos.

Este estudio demostró que la supresión de $\mathrm{HCl}$ se asoció a neumonía adquirida en el hospital. El aumento del riesgo alcanzó niveles de importancia en el uso de IBP, pero no en los antiH2. También se demostró relación con infección por $\mathrm{C}$. difficile adquirida en el hospital por el uso del tratamiento profiláctico (무).

Dada la escasez de pruebas directas que confirmen el beneficio de la supresión de $\mathrm{HCl}$ y la relación demostrada con infecciones oportunistas, no se recomienda la supresión de ácido para la prevención primaria de rutina de SDA en pacientes en unidades médicoquirúrgicas que no tengan factores de riesgo graves de SDA por LMRE (ㅁ).

- Profilaxis para sangrado gastrointestinal en pacientes críticamente enfermos, hospitalizados en $\mathrm{UCl}$ : en los pacientes internados en la $\mathrm{UCl}$, las úlceras gastrointestinales por estrés tienen una gran incidencia. Una gran mayoría de pacientes críticamente enfermos requiere profilaxis para la prevención primaria del sangrado por úlceras (15). La profilaxis no puede reducir el riesgo de hemorragia a cero, pero cuanto mayor es el riesgo de hemorragia, mayor es el beneficio esperado de la profilaxis (13). En base a estudios realizados por médicos, enfermeras y metodólogos de la Revista Médica Británica (BMJ, por sus siglas en inglés), se considera agrupar a los pacientes de riesgo de SDA en cuatro categorías: riesgo bajo, riesgo moderado, riesgo alto y riesgo más alto.

- Bajo riesgo: estado crítico sin ningún factor de riesgo asociado, insuficiencia hepática aguda, uso de corticosteroides $\mathrm{O}$ inmunosupresores, uso de anticoagulantes, cáncer y pacientes de género masculino.

- Moderado riesgo: ventilación mecánica asistida, nutrición enteral, shock, sepsis y lesión renal aguda.

- Alto riesgo: coagulopatía y asociar dos o más factores de riesgo moderado.

- Muy alto riesgo: ventilación mecánica sin nutrición enteral, hepatopatía crónica.

Los pacientes que tienen bajo riesgo presentan una probabilidad de sangrado de $1-2 \%$, los de moderado riesgo de 2$4 \%$, los de alto riesgo de $4-8 \%$ y los de muy alto riesgo de $8-10 \%$.

Se recomienda utilizar profilaxis para la supresión de ácido en pacientes con alto riesgo de hemorragia gastrointestinal (4\% o más). Se sugiere la utilización de un IBP, aunque el uso de antiH2 no se descarta como una opción razonable.

Para las personas con 1-2\% de riesgo de SDA clínicamente importante, puede que no haya diferencias significativas entre IBP y los antiH2. Sin embargo, para los pacientes con un riesgo de $4-10 \%$ de 
SDA clínicamente importante, los IBP pueden reducir el riesgo de sangrado más eficazmente que los antiH2.

El mejor agente específico es incierto. El pantoprazol, omeprazol, lansoprazol, esomeprazol y rabeprazol fueron los IBP más utilizados. La ranitidina y la famotidina fueron los antiH2 con más uso. Todos se consideran opciones adecuadas sin una evidencia clara de superioridad de uno por sobre otro entre la misma familia de medicamentos.

- Dosis y duración del tratamiento: por lo general, los IBP se recetan una vez al día y los antiH2 dos o tres veces al día. Ambos pueden administrarse por vía intravenosa o enteral, y no hay evidencia que sugiera que la vía de administración altere la eficacia (13).

La mayoría de los expertos consideran interrumpir la profilaxis después del alta de la $\mathrm{UCl}$, o cuando el paciente presente mejoría clínica y se resuelva el factor de riesgo de SDA por LMRE.

Sin embargo, en la literatura se evidencia la falta de criterios que indiquen cuándo es seguro suspender la supresión de $\mathrm{HCl}$.

Así mismo, no se evidencia en la literatura, una recomendación clara sobre la dosificación adecuada para la profilaxis del SDA por LMRE. Se recomienda en este caso empezar a titular el medicamento elegido, de menor a mayor dosis dependiendo de la aparición o no de signos de SDA.

\section{MORTALIDAD}

Existe incertidumbre sobre el impacto de los IPB y los antiH2 sobre el riesgo de mortalidad. No se ha logrado evidenciar cuál de los agentes presenta mejor tasa de sobrevida por sobre el otro. Así mismo, no se ha documentado diferencia en mejoría del riesgo de adquirir neumonía o infección por C. difficile dependiendo del uso entre estos medicamentos

\section{CONCLUSIONES}

- Las úlceras gástricas debidas a estrés fisiológico presentan una fisiopatología multifactorial, siendo el exceso de producción de $\mathrm{HCl}$ su desencadenante principal.

- La incidencia y prevalencia del sangrado digestivo superior es proporcional a nivel de estrés fisiológico que cursa el paciente, por lo cual es muy común encontrarlo en pacientes internados en la $\mathrm{UCl}$.

- La esofagogastroduodenoscopia es el estudio diagnóstico de elección ante la sospecha de úlceras gástricas 0 presencia de SDA. Así mismo se pueden brindar varias opciones terapéuticas con su uso, por lo cual presenta ventaja por sobre otros métodos diagnósticos.

- Los factores de riesgo que más se relacionaron con LMRE fueron: en pacientes sin enfermedad crítica el uso de anticoagulantes y clopidogrel, en pacientes críticamente enfermos la coagulopatía y la ventilación mecánica asistida.

- La Endoscopia por Video Cápsula es un

método seguro de diagnóstico, que cuenta con pocos efectos adversos con su uso. El costo y la disponibilidad del equipo, la falta de experiencia en el uso de este dispositivo y la falta del personal 
capacitado para su manejo son barreras importantes en la implementación global de la endoscopia con videocápsula.

- Se recomienda el uso profiláctico de medicamentos supresores del $\mathrm{HCl}$, en pacientes que presenten riesgo moderado, alto y muy alto de SDA por úlceras gástricas.

- En pacientes hospitalizados que no se encuentran críticamente enfermos, el uso de medicamentos profilácticos contra sangrado gastrointestinal no ha demostrado beneficio significativo en la morbi-mortalidad del paciente y se ha asociado con la aparición de infecciones oportunistas.

- En pacientes que presentan un $10 \%$ de riesgo de sangrado por úlcera por estrés, los Inhibidores de la Bomba de Protones han demostrado mayor efectividad en prevenir el sangrado que los Antagonistas de los Receptores de Histamina.

- Entre medicamentos de la misma familia (anti H2 o IBP) no se ha demostrado superioridad efectiva de un componente por sobre otro, para el tratamiento y prevención de las úlceras gástricas por estrés.

- No se recomienda el lavado nasogástrico como único método diagnóstico de sangrado gastrointestinal alto de origen ulceroso.

- No se cuenta con una guía clara para la suspensión del tiempo de uso de la profilaxis gástrica. Se recomienda descontinuar la medicación cuando el paciente egrese de la $\mathrm{UCl}$ o haya resuelto los factores de riesgo de úlcera gástrica por estrés.

- No se ha demostrado que se deba aumentar la dosis o frecuencia de los medicamentos profilácticos dependiendo del porcentaje de riesgo de sangrado que presente el paciente. Por lo cual se recomienda iniciar a dosis basal, titulando dependiendo de la clínica desarrollada por el paciente 0 encontrada en estudios complementarios.

\section{REFERENCIAS}

1. A.Krag, M, Perner Møller M. Profilaxis de úlcera por estrés en pacientes en unidad de cuidados intensivos. Revistas Ovid. 2016;(Volumen 22) p.15

2. Robertson M, Majumdar A, Boyapati R, Chung W. Risk stratification in acute upper GI bleeding: comparison of the AIMS65 score to the Glasgow-Blatchford and Rockall scoring systems. GIE: Gastrointestinal Endoscopy [Internet]. 2015 [citado el 24 Julio del 2020]; (6):3. Disponible en: https://www.giejournal.org/article/S0016$\underline{5107(15) 03037-0 / f u l l t e x t}$

3. Cook D, Guyatt G. Prophylaxis against Upper Gastrointestinal Bleeding in Hospitalized Patients. The New England Journal of medicine [Internet]. 2018 [citado el 24 julio del 2020];(26):2506-2514.Disponible en: https://doi.org/10.1056/NEJMra1605507

4. Vaki N. Enfermedad ulcerosa péptica: epidemiología, etiología y patogénesis. [Internet]. UpToDate. 2018 [citado el 24 Julio del 2020]. Disponible en: https://www-uptodate-com.binasss.idm.oclc.org/contents/peptic-ulcer- 
disease-epidemiology-etiology-andpathogenesis? search=sangrado $\% 20$ digestivo $\% 20$ alto\&topicRef $=2644 \&$ source $=$ see link

5. Siddiqui AH, Farooq U, Siddiqui F. Curling Ulcer. [Updated 2020 Apr 22]. In: StatPearls [Internet]. Treasure Island (FL): StatPearls Publishing; 2020 Jan-. Available from: https://www.ncbi.nlm.nih.gov/books/NBK482347/

6. Newberry C, Siddique S, Ahmad N, Metz D, Mehta S. BINASSS - Acceso a bases de datos [Internet]. Wwwclinicalkey-es.binasss.idm.oclc.org. 2020 [citado el 25 Julio 2020]. Disponible en: https://www-clinicalkeyes.binasss.idm.oclc.org/\#!/content/journal/1-s2.0-S0016508517310326

7. Sleisenger y Fordtran. Enfermedades digestivas y hepáticas, 10. ${ }^{a}$ edición. 10th ed. Feldman, Feldman \& Lawrence S. Friedman \& Lawrence J. Brandt. 2018. p 316

8. Siddiqui F, Ahmed M, Abbasi S, et al. Gastrointestinal Bleeding in Patients With Acute Respiratory Distress Syndrome: A National Database Analysis. J Clin Med Res. 2019;11(1):42-48. https://doi.org/10.14740/jocmr3660

9. Barletta, J. F., Bruno, J. J., Buckley, M. S., \& Cook, D. J. (2016). Stress Ulcer Prophylaxis. Critical care medicine, 44(7), 1395-1405. [citado el 16 de Agosto 2020]. https://doi.org/10.1097/CCM.0000000000001872

10. Wilkins T, Wheeler B \& Carpenter M. (2020). Upper Gastrointestinal Bleeding in Adults: Evaluation and Management. American Family Physician, 101(5), 294-300. [citado el 16 de Agosto 2020]. Disponible en: http://ovidsp.ovid.com/ovidweb.cgi?T=JS\&PAGE=reference\&D=medl\&NEWS=N\&AN=32109037.

11. Valle B, Díaz J, López J, Calderón C. Evaluación de la prescripción profiláctica de omeprazol y ranitidina mediante la identificación de factores de riesgo de sangrado gastrointestinal. Rev Colomb Cienc Quím Farm. 2017;(Vol. 46(1)). https://doi.org/10.15446/rcciquifa.v46n1.67289

12. Weinhouse G. Úlceras por estrés en la unidad de cuidados intensivos: diagnóstico, manejo y prevención [Internet]. Up to Date. 2020 [citado11 Agosto 2020]. Disponible en: https://www-uptodatecom.binasss.idm.oclc.org/contents/stress-ulcers-in-the-intensive-care-unit-diagnosis-management-andprevention.

13. Liu L, Siemieniuk R, Reintam Blaser A. Profilaxis de hemorragia gastrointestinal para pacientes críticamente enfermos: una práctica clínica guía. The British Medicine Journal. 2020.

14. Liu Y, Li D, Wen, A. Profilaxis farmacológica de la úlcera por estrés en pacientes que no acuden a la UCl: una revisión sistemática y un metanálisis en red de ensayos controlados aleatorios [Internet]. Clinical Key. 2020 [citado 7 agosto 2020]. Disponible en: https://www-clinicalkey-es.binasss.idm.oclc.org.

15. Awadalla, Mohanad, Desimone, Michael \& Wassef, Wahid. (2019). Updates on management of nonvariceal upper gastrointestinal bleeding. Current Opinion in Gastroenterology, 35, 517-523. [citado el 16 de Agosto2020]. https://doi.org/10.1097/MOG.0000000000000582 